\title{
Early stage resectable non-small cell lung cancer: is neoadjuvant immunotherapy the right way forward?
}

\author{
Rita Chiari ${ }^{1}$, Angelo Sidoni ${ }^{2}$, Giulio Metro ${ }^{1}$ \\ ${ }^{1}$ Medical Oncology, Santa Maria della Misericordia Hospital, Azienda Ospedaliera di Perugia, Perugia, Italy; ${ }^{2}$ Section of Anatomic Pathology and \\ Histology, Department of Experimental Medicine, University of Perugia Medical School, Perugia, Italy \\ Correspondence to: Giulio Metro, MD. Staff Physician, Medical Oncology, Santa Maria della Misericordia Hospital, Azienda Ospedaliera di Perugia, \\ via Dottori, 1, 06156, Perugia, Italy. Email: giulio.metro@yahoo.com. \\ Provenance: This is an invited Editorial commissioned by the Section Editor Chunlin Ou (Cancer Research Institute of Central South University, \\ Changsha, China). \\ Comment on: Forde PM, Chaft JE, Smith KN, et al. Neoadjuvant PD-1 blockade in resectable lung cancer. N Engl J Med 2018;378:1976-86.
}

Submitted Aug 09, 2018. Accepted for publication Sep 28, 2018.

doi: $10.21037 /$ jtd.2018.10.22

View this article at: http://dx.doi.org/10.21037/jtd.2018.10.22

Lung cancer represents the major cause of cancer-related mortality in countries with a high degree of socioeconomic development, with non-small cell lung cancer (NSCLC) accounting for approximately $85 \%$ of all primary lung cancers $(1,2)$. According to SEER registries, the rate of newly diagnosed NSCLC patients with localized (confined to primary site), or regional (spread to regional lymph nodes) diseases is $16 \%$ and $22 \%$, respectively (3). Unfortunately, even for these patients, who may potentially benefit from the use of local therapies (i.e., surgery and/or radiotherapy), the 5 -year survival is poor, being $56.3 \%$ for localized disease, and $29.7 \%$ for regional disease. Such a dismal prognosis mainly reflects the tendency these patients have to experience distant metastases over time.

Against this background, both neoadjuvant and adjuvant chemotherapy have been shown to improve survival of patients with early stage surgically resectable NSCLC, with no significant differences in terms of either disease-free or overall survival between the two strategies (4-6). Notwithstanding, in routine clinical practice, adjuvant chemotherapy is often preferred to the neoadjuvant approach, mainly because of the fear that, if treatment-related complications arise during neoadjuvant chemotherapy, surgery may be delayed to a point that is no longer feasible because of rapid tumor progression. On the other hand, there are a few issues that seem to favor the use of neoadjuvant versus adjuvant chemotherapy, including, but not limited to, early cure of micrometastatic disease, better chemotherapy drug delivery and tolerability, ability to assess sensitivity to treatment, and acquisition of prognostic information based on whether a major pathologic response ( $\leq 10 \%$ of residual viable tumor at pathologic examination) has occurred (7-9). According to these considerations, neoadjuvant chemotherapy should be regarded as an option, and this has been acknowledged by NCCN guidelines which clearly state that 'after surgical evaluation patients likely to receive adjuvant chemotherapy may be treated with induction chemotherapy as an alternative' (10).

In recent years, medical oncologists have been witnessing a revolution in the treatment of advanced NSCLC, owing to the introduction for clinical use of highly effective therapies such as cancer immunotherapy (11). Basically, immunotherapy acts by reverting the immune self-tolerance pathways through which the tumor avoids immune recognition and destruction. At the present time, immune checkpoint inhibitors (ICIs) are widely used monoclonal antibodies in this field. Specifically, ICIs' anti-tumor mechanism of action is based on modulation of T-cell function at two different levels: centrally, by blocking the inhibitory interaction between cytotoxic T-lymphocyte antigen 4 (CTLA-4) expressed on T-cells, and B7 expressed on the antigen presenting cell, or peripherally, by antagonizing the inhibitory interaction between the antiprogrammed cell death-1 (PD-1) expressed on T-cells, and anti-programmed cell death ligand-1 (PD-L1) or -2 (PD-L2), mainly expressed on tumor cells (12). As a 
result, disruption of the CTLA-4 or PD-1 axes activates immune system against malignant cells. Importantly, immunohistochemical expression of PD-L1 on tumor cell membrane has emerged as a biomarker of response to immunotherapy, particularly for PD-1 and PD-L1 inhibitors. To date, several ICIs have been approved for the treatment of advanced NSCLC (13). The antiPD-L1 agent atezolizumab and the anti- PD-1 drugs nivolumab and pembrolizumab are used in the second-line setting, with pembrolizumab reserved for tumors with a PD-L1 expression $\geq 1 \%$. More recently, pembrolizumab has also gained first-line approval for NSCLCs with a PD-L1 $\geq 50 \%$, while the anti-PD-L1 agent durvalumab has been approved as maintenance treatment after chemoradiotherapy for unresectable stage III disease. In addition, emerging data support the use of an ICI in the first-line treatment of advanced NSCLC, namely pembrolizumab with platinum-based chemotherapy or atezolizumab plus platinum-based chemotherapy and bevacizumab, the latter for non-squamous disease only. Finally an ICI combination of nivolumab plus the anti-CTLA-4 agent ipilimumab has been proven effective in NSCLCs with a high tumor mutation burden (TMB), which reflects the total number of non-synonymous mutations per coding area of a tumor genome. In fact, in line with the fact that a high TMB may render the tumor more immunogenic, it has been associated with increased sensitivity to ICIs in general.

The important results obtained with immunotherapy in advanced NSCLC have prompted the investigation of ICIs also in earlier stages of the disease. A recent study evaluated the immune microenvironment in tumors obtained from patients with early stage NSCLC, and found that a significant proportion of tumor-infiltrating T-cells expressed PD-1, while a higher PD-L1 expression was noted in tumors compared with adjacent normal lung tissue (14). Even more importantly, anti-PD-1 treatment resulted into improved survival in orthotopic murine models of KRASmutant (G12D) early stage NSCLC. Taken together, these data suggest that the immune-suppressive PD-1 axis is activated early in NSCLC, where it may function as a therapeutic target, thus providing a rationale for the use of ICIs in early stage disease. Theoretically, ICIs could be more effective when used as neoadjuvant rather adjuvant treatment, as the presence of larger masses with T-cell infiltration may favor the establishment of an adaptive antitumor immune response. Importantly, induction of an immune response before surgery will result into immune memory, potentially leading to a durable and robust protection against cancer recurrence over a long term.

Recently, Forde and colleagues reported on a study (NCT02259621) employing nivolumab as neoadjuvant treatment of early stage NSCLC (15). This was a singlearm trial in which NSCLC patients who were deemed to be surgically resectable received two pre-operative doses of nivolumab at $3 \mathrm{mg} / \mathrm{kg}$ every 2 weeks. Primary end-points were safety and feasibility of resection, while secondary end-points were radiological and pathologic response to treatment. Interestingly, there were also a few exploratory end-points, including immunologic, genomic, and pathologic correlates of response in blood and tumor. Overall, 22 stage I-IIIA patients were enrolled, with 21 being eligible for inclusion in the study. Only one grade $\geq 3$ toxicity was observed in one patient, namely grade 3 pneumonia, which, however, did not prevent an uncomplicated surgical resection after one dose of nivolumab. On the other hand, nivolumab did not result into any surgical delays. Radiographic partial responses occurred in $10 \%$ of patients ( 2 of 21 patients), while major pathologic response at surgery were documented in $45 \%$ of individuals ( 9 out of 20 resected patients). Of note, pathologic responses were observed in both PD-L1positive and PD-L1-negative tumors, while whole exome sequencing, which could be carried out only in 11 patients with resected tumors for whom pre-treatment cancer tissue was available, showed significantly higher mean levels of TMB in patients who experienced a major pathologic response, as well as an inverse correlation between TMB and residual viable tumor cells at pathologic examination. Finally, systemic immune responses that were evaluated by deep sequencing of the $\mathrm{T}$ cell receptor gene (TCR) in 9 patients showed a higher frequency of shared TCR sequences in the tumor and blood compartments for those patients who had a major pathologic response, with 8 out of 9 individuals having peripheral expansion of multiple T-cell clones in the peripheral blood. This phenomenon indicates that the formation of $\mathrm{T}$-cell clones committed against the tumor can expand to other tissues in order to fight micrometastatic disease. The importance of this study is that it provides evidence that a short course of preoperative immunotherapy does not jeopardize a safe and timely surgery in patients with early-stage NSCLC, while it may translate into a relevant percentage of major pathologic response. Of note, it also suggested that neoadjuvant immunotherapy leads to early induction of an adaptive antitumor immunity which may be responsible for preventing distant metastases. However, confirmatory studies are 
needed, as the trial by Forde $e t$ al. enrolled very few patients. Also, the short follow-up and the absence of randomization does not allow to draw exhaustive conclusions with regard to the impact of neoadjuvant immunotherapy on diseasefree and overall survival.

Recently, the preliminary results of a study (NCT02927301) investigating neoadjuvant atezolizumab for early stage NSCLCs have been reported (16). This study is composed by 2 parts: in part 1, approximately 180 patients are subjected to 2 doses of pre-operative atezolizumab, with a pre-specified interim analysis planned after completion of neoadjuvant atezolizumab in the first 30 patients. Part 2 is exploratory, and evaluates adjuvant atezolizumab for up to 12 months in part 1 patients who have demonstrated clinical benefit. Standard of care chemotherapy with or without radiation is allowed prior to adjuvant atezolizumab in part 2. The early reported results of part 1 refer to the first 37 enrolled patients and documented a major pathologic response in $19 \%$ of individuals (6 out of 32 evaluable patients). Importantly, only one severe treatment-related adverse event occurred in the overall population, consisting of grade 3 dyspnea. In conclusion, these preliminary results seemed to confirm the activity of a short course of neoadjuvant immunotherapy in resectable NSCLCs, also highlighting the fact that surgical resection can be accomplished in a safe manner with no major delays. This study is ongoing, and final results will help clarify the role of perioperative atezolizumab in a large NSCLC population.

Other ongoing studies are also looking at the combination of chemotherapy plus immunotherapy in order to further improve the rate of pathologic response. A recent phase 2 trial (EudraCT 2016-003732-20) employing 3 cycles of neoadjuvant nivolumab + carboplatin and paclitaxel for resectable stage IIIA-N2 NSCLC patients reported a complete pathologic response of $60 \%$ (13 patients), with a major pathologic response of $18 \%$ (4 patients) in the first 22 resected patients (17). Another single arm study (NCT02716038) is evaluating the combination of atezolizumab + nab-paclitaxel and carboplatin for four cycles prior to surgery in stage IB-IIIA NSCLCs (18). Very early results on 14 patients have been presented. Overall, $78.6 \%$ of patients (11 out of 14 ) underwent a safe surgical resection, with 3 patients who were found not to be surgically resectable at the time of surgery. Of note, of those who were resected, a complete and major pathologic response occurred in $27.3 \%$ ( 3 patients) and $63.6 \%$ individuals (7 patients). Although preliminary, the results of these two phase 2 studies suggest that the addition of chemotherapy to immunotherapy leads to an increase in terms of major pathologic response, with the chance of achieving a complete pathologic response in up to one fourth of patients.

The above mentioned studies support the role for neoadjuvant immunotherapy in surgically resectable NSCLC. However, we are still in an early stage of clinical development, and there are several pending questions yet to be answered. First, what is the best way to integrate immunotherapy in the neoadjuvant treatment. Options include immunotherapy as single agent, immunotherapy plus chemotherapy or immunotherapy combinations [e.g., anti-PD-(L)1 plus anti-CTLA-4]. Second, what is the optimal duration of neoadjuvant treatment and what is the contribution of adjuvant immunotherapy in patients who benefit from pre-operative treatment. Third, whether major pathologic response could represent a surrogate end-point for survival in this setting is yet to be answered. Finally, it would be important to identify upfront who are the patients that may benefit from neoadjuvant immunotherapy. With regard to this, the assessment of PD-L1 and/or TMB status on tumor biopsies prior to initiation of neoadjuvant treatment may be helpful in selecting the right candidates to treatment. At the same time, patients whose tumor bears an actionable mutation (i.e., EGFR, ALK, and ROS1) should be excluded, based on the evidence that oncogene-addicted tumors are generally poor responders to immunotherapy (19). However, comprehensive molecular genotyping could be problematic if limited amounts of tumor DNA are available, as it is the case after a conventional or fine needle biopsy in patients who are staged with resectable disease. This, in turn, may further limit the clinical applicability of neoadjuvant immunotherapy. In the next future it can be envisioned that TMB assessment on circulating tumor DNA might replace tumor tissue in the identification of a molecular genotype that may predict response to immunotherapy, and prospective trials are ongoing in order to provide clinical validation of blood-based gene mutation testing (20-22).

In conclusion, at the present time, we still do not know whether neoadjuvant immunotherapy will benefit these patients in terms of disease-free and, ultimately, overall survival. Phase 3 studies of neoadjuvant immunotherapy are underway (Table 1). Importantly, all of them include a number of correlative studies on biomarkers of response among their secondary end-points, that will definitely help 
Table 1 Select ongoing phase 3 trials on neoadjuvant immunotherapy for early stage resectable non-small cell lung cancer patients

\begin{tabular}{lll}
\hline Study (ClinicalTrials.gov identifier) & Design & Stages \\
\hline CheckMate 816 (NCT02998528) & Nivolumab + ipilimumab vs. & IB ( $\geq 4 \mathrm{~cm})$-IIIA according to $7^{\text {th }}$ Ed \\
& Nivolumab + platinum doublet vs. & \\
& Platinum doublet & II-IIIA and select stage IIIB (T3N2 only) \\
IMpower030 (NCT03456063) & Atezolizumab + platinum doublet vs. & according to $8^{\text {th }}$ Ed \\
& Platinum doublet & \\
KEYNOTE-671 (NCT03425643) & Adjuvant atezolizumab in the experimental arm & IIB-IIIA according to $8^{\text {th }}$ Ed \\
& Pembrolizumab + platinum doublet vs. & \\
& Platinum doublet & Adjuvant pembrolizumab in the experimental arm \\
\hline
\end{tabular}

identify those tumors who are likely to be immunosensitive, and for whom immunotherapy can lead to a substantial increase in the cure rate.

\section{Acknowledgements}

None.

\section{Footnote}

Conflicts of Interest: The authors have no conflicts of interest to declare.

\section{References}

1. Rafiemanesh H, Mehtarpour M, Khani F, et al. Epidemiology, incidence and mortality of lung cancer and their relationship with the development index in the world. J Thorac Dis 2016;8:1094-102.

2. Wong MCS, Lao XQ, Ho KF, et al. Incidence and mortality of lung cancer: global trends and association with socioeconomic status. Sci Rep 2017;7:14300.

3. SEER Cancer Stat Facts: Lung and Bronchus Cancer [cited 2018 Aug 8]. Available online: https://seer.cancer. gov/statfacts/html/lungb.html

4. Burdett S, Pignon JP, Tierney J, et al. Adjuvant chemotherapy for resected early-stage non-small cell lung cancer. Cochrane Database Syst Rev 2015;(3):CD011430.

5. NSCLC Meta-analysis Collaborative Group. Preoperative chemotherapy for non-small-cell lung cancer: a systematic review and meta-analysis of individual participant data.
Lancet 2014;383:1561-71.

6. Lim E, Harris G, Patel A, et al. Preoperative versus postoperative chemotherapy in patients with resectable non-small cell lung cancer: systematic review and indirect comparison meta-analysis of randomized trials. J Thorac Oncol 2009;4:1380-8.

7. Felip E, Rosell R, Maestre JA, et al. Preoperative chemotherapy plus surgery versus surgery plus adjuvant chemotherapy versus surgery alone in early-stage nonsmall-cell lung cancer. J Clin Oncol 2010;28:3138-45.

8. Chaft JE, Dunphy M, Naidoo J, et al. Adaptive neoadjuvant chemotherapy guided by (18)F-FDG PET in resectable non-small cell lung cancers: the NEOSCAN trial. J Thorac Oncol 2016;11:537-44.

9. Hellmann MD, Chaft JE, William WN Jr, et al. Pathological response after neoadjuvant chemotherapy in resectable non-small-cell lung cancers: proposal for the use of major pathological response as a surrogate endpoint. Lancet Oncol 2014;15:e42-50.

10. NCCN Clinical Practice Guidelines in Oncology: NonSmall Cell Lung Cancer - [cited 2018 Aug 8]. Available online: https://www.nccn.org/professionals/physician_gls/ pdf/nscl.pdf

11. Toschi L, Rossi S, Finocchiaro G, Santoro A. Nonsmall cell lung cancer treatment (r)evolution: ten years of advances and more to come. Ecancermedicalscience 2017;11:787.

12. Ribas A, Wolchok JD. Cancer immunotherapy using checkpoint blockade. Science 2018;359:1350-5.

13. Brahmer JR, Govindan R, Anders RA, et al. The Society for Immunotherapy of Cancer consensus statement on immunotherapy for the treatment of non-small cell lung 
cancer (NSCLC). J Immunother Cancer 2018;6:75.

14. Markowitz GJ, Havel LS, Crowley MJ, et al. Immune reprogramming via PD-1 inhibition enhances early-stage lung cancer survival. JCI Insight 2018;3. [Epub ahead of print].

15. Forde PM, Chaft JE, Smith KN, et al. Neoadjuvant PD-1 blockade in resectable lung cancer. N Engl J Med 2018;378:1976-86.

16. Rusch VW, Chaft JE, Johnson B, et al. Neoadjuvant atezolizumab in resectable non-small cell lung cancer (NSCLC): Initial results from a multicenter study (LCMC3). J Clin Oncol 2018;36:abstr 8541.

17. Provencio-Pulla M, Nadal-Alforja E, Cobo M, et al. Neoadjuvant chemo/immunotherapy for the treatment of stages IIIA resectable non-small cell lung cancer (NSCLC): A phase II multicenter exploratory study-NADIM studySLCG. J Clin Oncol 2018;36:abstr 8521.

18. Shu CA, Grigg C, Chiuzan C, et al. Neoadjuvant

Cite this article as: Chiari R, Sidoni A, Metro G. Early stage resectable non-small cell lung cancer: is neoadjuvant immunotherapy the right way forward? J Thorac Dis 2018;10(Suppl 33):S3890-S3894. doi: 10.21037/jtd.2018.10.22 atezolizumab + chemotherapy in resectable non-small cell lung cancer (NSCLC). J Clin Oncol 2018;36:abstr 8521.

19. Metro G, Di Maio M. Cons: should immunotherapy be incorporated in the treatment of oncogene-driven lung cancer? Transl Lung Cancer Res 2018;7:S290-S293.

20. Khagi Y, Goodman AM, Daniels GA, et al. Hypermutated Circulating Tumor DNA: Correlation with Response to Checkpoint Inhibitor-Based Immunotherapy. Clin Cancer Res 2017;23:5729-36.

21. Gandara DR, Paul SM, Kowanetz M, et al. Bloodbased tumor mutational burden as a predictor of clinical benefit in non-small-cell lung cancer patients treated with atezolizumab. Nat Med. 2018;24:1441-8.

22. Velcheti V, Kim ES, Mekhail T, et al. Prospective clinical evaluation of blood-based tumor mutational burden (bTMB) as a predictive biomarker for atezolizumab (atezo) in 1L non-small cell lung cancer (NSCLC): Interim B-F1RST results. J Clin Oncol 2018;36:abstr 12001. 